Article

\title{
Developing an IoT-Enabled Cloud Management Platform for Agricultural Machinery Equipped with Automatic Navigation Systems
}

\author{
Fan Zhang ${ }^{1,2}$, Wenyu Zhang ${ }^{1,2}$, Xiwen Luo ${ }^{1,2}$, Zhigang Zhang ${ }^{1,2, *}$, Yueteng Lu ${ }^{1,2}$ and Ben Wang ${ }^{1,2}$ \\ 1 Key Laboratory of the Ministry of Education of China for Key Technologies for Agricultural Machine and \\ Equipment, South China Agricultural University, Guangzhou 510642, China; zf1024@stu.scau.edu.cn (F.Z.); \\ zhangwenyu@scau.edu.cn (W.Z.); xwluo@scau.edu.cn (X.L.); luyt@stu.scau.edu.cn (Y.L.); \\ wangben@stu.scau.edu.cn (B.W.) \\ 2 College of Engineering, South China Agricultural University, Guangzhou 510642, China \\ * Correspondence: zzg208@scau.edu.cn; Tel.: +86-20-38676975
}

Citation: Zhang, F.; Zhang, W.; Luo,

X.; Zhang, Z.; Lu, Y.; Wang, B.

Developing an IoT-Enabled Cloud

Management Platform for

Agricultural Machinery Equipped

with Automatic Navigation Systems.

Agriculture 2022, 12, 310.

https://doi.org/10.3390/

agriculture 12020310

Academic Editor: Andrea Colantoni

Received: 6 January 2022

Accepted: 16 February 2022

Published: 21 February 2022

Publisher's Note: MDPI stays neutral with regard to jurisdictional claims in published maps and institutional affiliations.

Copyright: (C) 2022 by the authors. Licensee MDPI, Basel, Switzerland. This article is an open access article distributed under the terms and conditions of the Creative Commons Attribution (CC BY) license (https:// creativecommons.org/licenses/by/ $4.0 /)$.

\begin{abstract}
Smart farming uses advanced tools and technologies such as intelligent agricultural machines, high-precision sensors, navigation systems, and sophisticated computer systems to increase the economic benefits of agriculture and reduce the associated human effort. With the increasing demands of individualized farming operations, the internet of things is a crucial technique for acquiring, monitoring, processing, and managing the agricultural resource data of precision agriculture and ecological monitoring domains. Here, an internet of things-based system scheme integrating the most recent technologies for designing a management platform for agricultural machines equipped with automatic navigation systems is proposed. Various agricultural machinery cyber-models and their corresponding sensor nodes were constructed in a pre-production phase. Three key enabling technologies-multi-optimization of agricultural machinery scheduling, development of physical architecture and software, and integration of the controller-area-network with a mobile network-were addressed to support the system scheme. A demonstrative prototype system was developed and a case study was used to validate the feasibility and effectiveness of the proposed approach.
\end{abstract}

Keywords: smart farming; internet of things; intelligent agricultural machines; management platform; agricultural machinery scheduling

\section{Introduction}

The mass real-time demands of resource allocation as well as data processing in agriculture are currently placing new requirements on precision agriculture [1,2]. Internet of things (IoT) and cloud computing involve the basic management tasks not only of farming processes, but also of visual data related to crop resources and growth environments [3,4]. They are propelling the rapid development of smart farming. The farm management information system (FMIS), which includes the functions of data collection and analysis, decision making, and controlling variable rates, improves agricultural production and reduces negative impacts on the environment [5]. However, owing to the individual requirements of system construction, a customized operational system for each farm presents various characteristics [6], including differences in various crops, the constraints of field conditions, the diversity of machine capacities, and specificities of pathways. Therefore, how to connect the FMIS to real smart farming has become a major issue.

In the main activity of arable farming, traditional agricultural equipment can only communicate with farmer operators, which brings many challenges to the development of smart farming, such as the efficiency and stability of transmission [7], and the difficult integration between current communication technology (ICT) devices and farm enterprises. Even though a great many researchers have studied the problems of agricultural machinery 
management and resource allocation in FIMS, this information cannot directly contribute to agricultural machinery, which leads to the farmer having to pay additional fees for the resources consumed in the process of distributing and operating operational assignments for agricultural machinery throughout the farm work. Therefore, it is essential to develop a new system to bridge the communication gap between agricultural machinery and FMIS, and maximize resource performance to help farmers digitally transform the management of their farms and agricultural machinery [8].

Fundamental to this transformation is the rapid development of some technologies, including IoT, information technology and ICT, and agricultural autonomous driving techniques. In terms of IoT and ICT, they have played important roles in the connectivity of FMIS and have usually been used to collect real-time data on facilities and crops that are part of the farms. With regards to agricultural autonomous driving techniques, this is realized by transforming the chassis of traditional agricultural machinery and installing automatic navigation operational systems, which not only promote the emergence of autonomous agricultural machinery (AAM), but also provide key support for the implementation of smart farming in China [9-11].

AAM includes automatic navigation operational tractors, unmanned aerial vehicles, agricultural robots, etc., which are used to conduct and support different services and tasks that are assigned by the farm managers, tractor operators, or researchers. However, some new demands for these devices have been raised when used on farms. One is an urgent need to build an IoT-enabled management platform for these new machines to acquire the basic data of measurements taken in the field through simple-to-use methods [12,13]. Another is that the two key decision problems-dynamic work-planning and decisionmaking-should be addressed so that the emerging AAM can be controlled and operated in the field in the most efficient manner [14,15].

To meet the above requirements, an IoT-enabled autonomous agricultural machinery management platform (AAMMP) scheme was introduced to perceive the current position of the agricultural machinery and provide motion recommendations based on the physical environment. The aim was to unify an IoT-based system to exchange data from realtime operating AAMs through communication. In addition, a dynamic optimization framework is proposed to decide when, where, and how the AAM should work to improve the work efficiency of the farm for a period of precise operation. Therefore, the main content for the AAMMP includes three steps. Firstly, this study will describe how the embedded system of AAM will be connected with the IoT-based platform to perceive real-time information and execute low-latency motions. Moreover, a novel decoupling algorithm based on a multi-object scheduling model is proposed to providing decisionmaking on scheduling, operational allocation, and path planning of AAM. Finally, an individualized IoT-based platform for agricultural machinery management was developed and an illustrative operational procedure is discussed using a case study of practical AAM operations.

The outline of this study is organized as follows. Section 2 is the literature review of related works and Section 3 describes the architecture and design logistics. Three key enabling technologies are illustrated in Section 4: multi-optimization and cloud computing, system development, and a communication and integration strategy. Section 5 presents a case study on the implementation using an AAMMP system. Finally, the conclusions are provided in Section 6.

\section{Related Works}

\subsection{IoT-Based System}

IoT technology has been widely used in FMIS to support the automation of data acquisition, monitoring, planning, decision making, and management of farm operations. Application scenarios for IoT using state-of-the-art sensors and devices have been investigated, such as monitoring climatic conditions to improve seed growth [16], controlling light intensity in greenhouse cultivation $[17,18]$, planning water-resource irrigation and 
proper utilization of water resources [19], and assessing the freshness levels of fruit [20]. When it comes to agricultural machinery management, IoT-based solutions for application are still in their early stages. Table 1 shows the sensors and networks, whether to integrate with the on-board motion controller of agricultural machinery, and the main functions of proposed system in previous studies.

Table 1. Comparison of proposed systems.

\begin{tabular}{ccccc}
\hline Reference & Sensors & Networks & Integration (Y/N) & Functions \\
\hline Chaudhary et al. [21] & GPS & GPRS, Bluetooth & N & Monitoring and management \\
Oksanen et al. [22] & GPS & 3G, CAN & Y & Remote monitoring \\
Zhang et al. [23] & GPS & GPRS, Wi-Fi, 4G & M & Monitoring and management \\
Li et al. [24] & GPS & CAN, RS232 & Y & Data acquisition and processing \\
Wei Fu [25] & GDS & GPRS & Monitoring and management \\
Wan-Soo Kim [26] & - & - & - & Operation service \\
\hline
\end{tabular}

Abbreviations: GPS, Global Positioning System; GPRS, General Packet Radio Service; CAN, Controller-AreaNetwork; N, No; Y, Yes.

AGCO, the most famous agricultural machinery company in America, has proposed a management system called the "connected farm service" for farm and agricultural machinery [21]. IoT-based field operation monitoring systems have been effectively implemented in harvesting operations [22]. Zhang designed an agricultural machinery service management system by installing remote monitoring terminals on the agricultural machinery to support low-cost and high-efficiency production for the company [23]. Li et al. used IoT technology to develop an agricultural machinery networking platform to achieve the provisions of regional agricultural operations monitoring and management, farmland status monitoring, and service functions [24]. Based on "internet + BDS + GIS" (BDS, BeiDou Navigation Satellite System; GIS, Geographic Information System) technology, an intelligent supervision system was proposed to monitor and manage farm machinery [25]. John Deere has developed three integrated systems to help manage the operation of agricultural machinery remotely, including the Machine Sync system, AutoTrac Vision, and AutoTrac RowSense system [26].

From Table 1, the sensors of the proposed systems are GPS (Global Positioning System) and BDS, which can be used to acquire the location information of agricultural machinery. As for the networks, some of them install GPS sensors in the agricultural machinery to acquire location information. Others propose the development of a new embedded system integrated with the on-board motion controller of agricultural machinery; thus, the CANbus network is used to integrate with on-board motion controllers of agricultural machinery, and then data is packaged by ISO 11783 protocol. All of these data are sent to IoT-based systems by wireless networks, such as GPRS (General Packet Radio Service), 3G/4G, Wi-Fi, and Bluetooth. Moreover, the functions of the proposed systems are mainly monitoring and management, while there are fewer reports on the result of integration and how to execute the operation optimization service. Therefore, this study will focus on constructing a unified AAMMP adapting to the various needs of farming operations, such as real-time monitoring, machinery scheduling, and operational distribution.

\subsection{Operations Assignment and Scheduling of Multiple Machines}

The operations assignment and scheduling of multiple machines are key elements of the AAMMP, and a reasonable work scheme can reduce the cost of the entire process and improve work efficiency. To maximize the total area operated by the sugarcane harvester, Pitakaso et al. [27] presented an ALNS metaheuristics approach to solve largescale problems such as harvester assignment and mechanical harvester routing problems. Cao et al. [28] studied the task assignment problem in multi-machine cooperative navigation and proposed the improved ant colony algorithm to operate multiple agricultural machines in a cooperative manner. A hybrid tabu search algorithm was proposed by He et al. [29] 
to optimize the wheat harvesting period and balance harvesting times among combineharvesters. Zhang et al. [30] proposed an improved multi-parental genetic algorithm to distribute multiple machines to execute multiple tasks sequentially in different farmlands. Aiming at solve the scheduling and allocation problems of farm machinery, a scheduling and allocating algorithm based on non-cooperative game theory was proposed to optimize the costs of economy and time.

Although researchers have made great achievements in the assignment and scheduling of multi-machine problems, there are still many practical constraints that need to be taken into consideration during the actual operational process. First, when building mathematical models for this optimization problem, some other factors should be addressed, such as the matching of job execution, the operational cycle, and the real-work efficiency of the agricultural machinery [28]. In addition, a dynamic regulatory strategy should be used during the operation of the agricultural machinery, because there might be exceptions to normal processing such as machinery break downs and emergency tasks that need to be prioritized [31]. Finally, the route selection for field coverage planning is also a vital function of the AAMMP. This is also a constraint that affects the work efficiency and make-span [32]. A route optimization algorithm can minimize the operating distance of a sugarcane harvester and maximize the sugarcane field harvest [27]. It can also improve field operations and reduce non-productive travel distance when applying liquid fertilizer [33]. Therefore, optimizing multiple agricultural machinery scheduling and pathways should be treated as a coupled optimization problem, and it is necessary to establish a proper method to accommodate both static allocation and dynamic adjustment for multi-machine scheduling problems.

This study used cooperative operations of agricultural machinery equipped with navigation systems as an implementation scenario and proposes an IoT-enabled management system. This system goes beyond offline task management and scheduling and allows either online monitoring or even dynamic optimization based on operational data acquired in real time.

\section{Methodology}

\subsection{Experimental Site}

The research was conducted at the Key Laboratory of the Ministry of Education for Key Technologies of Agricultural Machinery and Equipment in South China, South China Agricultural University (SCAU). The experimental site was located at the Zengcheng Teaching and Research Bases. The agricultural machines were made by LOVOL (WeiFang City, Shandong Province, China), and the automatic navigation system was self-developed and used to control the devices' motion and to acquire data from the agricultural machines.

\subsection{Design Method and System Architecture}

An AAMMP can be described as a set of automatic agricultural devices that are controlled and traced by predefined control instructions constrained by task diversity, varying environments, and different network connections. Here, we propose an IoT-based design approach to manage cooperative operations for multiple agricultural machines on arable farming.

As shown in Figure 1, when an operational task is assigned, its related demands (e.g., machinery assembly, path planning, control scheme, and work efficiency) are parameterized and sent to the cloud platform for further operational scheduling. The static execution solution is generated by the multiple optimization method executed through cloud computing. However, several emergency tasks may emerge due to unpredicted situations during the task processing, including bad weather and equipment malfunctions. For example, a working tractor cooperating with other tractors may be transferred to another field to work, interrupting the previous operational plan and resulting in previous tasks being reconfigured. Therefore, these priorities need to be addressed by a dynamic result generated by coupling the optimization of the previous job and the next urgent task. Thus, 
the dynamic execution solution will be constrained by the static results because they can directly affect parameters and iterations of the coupling optimization method. After that, by pursuing the balance and stability of the static and dynamic scheduling plan, the control directions can be created and sent to the on-board system of the agriculture machinery through a mobile network.

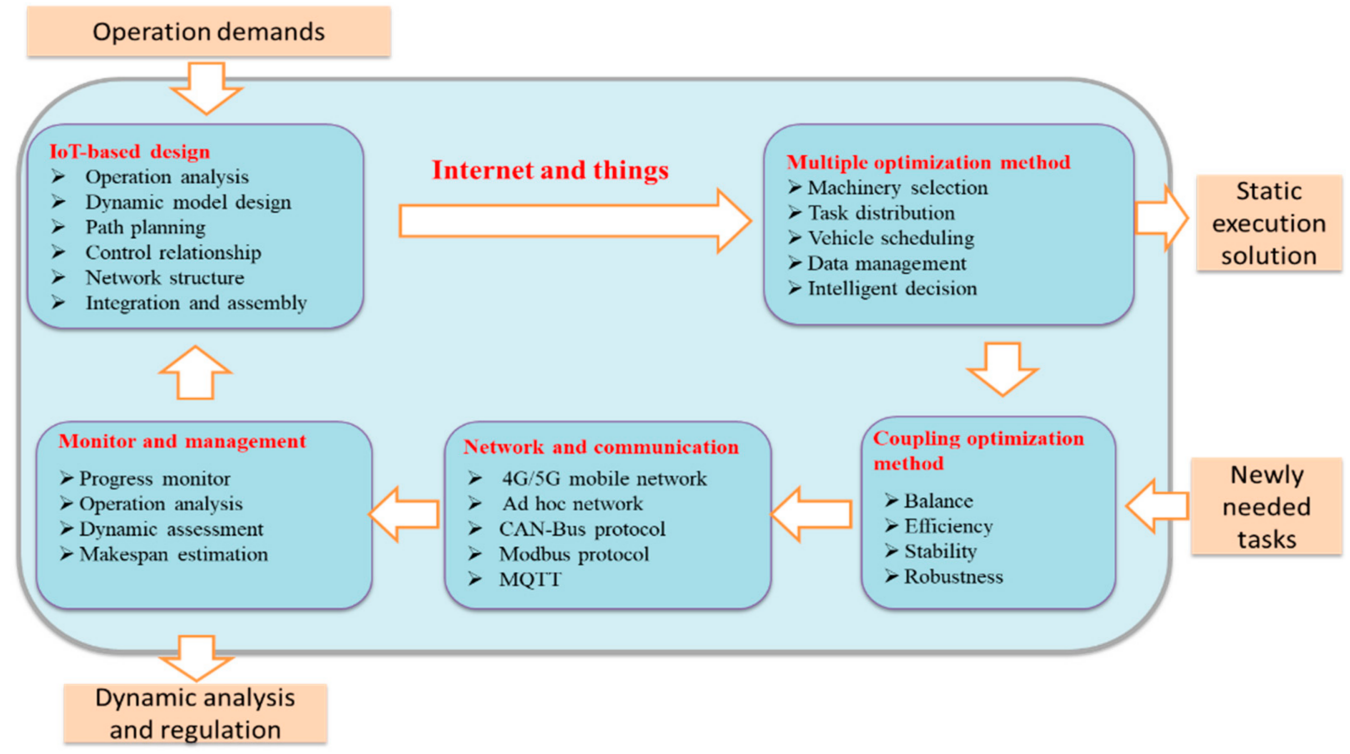

Figure 1. IoT-based design approach logic.

Because AAMMPs must address dynamic changes in operational tasks and locations, variation and changes in operations are conventionally associated with the sustained improvement of dynamic execution schemes. Conventional solutions only focus on the scheduling of static tasks because of the instability or insufficiency of the information communication, this makes it difficult to make real-time adjustments in assignment during agricultural production. The proposed IoT-based design approach intended is to improve the capability of the dynamic replanning scheme for the AAMMP before the start of production, thereby making changes in machinery scheduling after operational implementation quicker and less costly.

The key part of the proposed AAMMP structure is the cloud layer, which is used to control various agricultural machines and decides all the functionalities of the system. Figure 2 shows the architectural diagram of the AAMMP, which can be classified into two entities: the physical item (or things) and software. The physical item consists of perception and network layers, including embedded devices, sensors, data transmission modules, routers, and gateways. Because all the machines have embedded computing and communication capabilities, they can perceive the environment through a navigation or machine vision system and then interact with each other using direct or indirect communications to ensure high-quality cooperation. Direct communications can be implemented by an ad hoc network such as mesh topology, whereas indirect communication can occur with a mobile gateway or base station through a star topology. For the software, business and service layers mainly concentrate on data management and business control separately. Between the two items, the cloud platform plays the key role of intelligent brain and manager, which can control the physical entities in the background businesses and also manage the upstream data and service applications. Finally, customers and users can monitor and manage agricultural machinery in the whole farm through visiting Web client service or App (Application) service. 


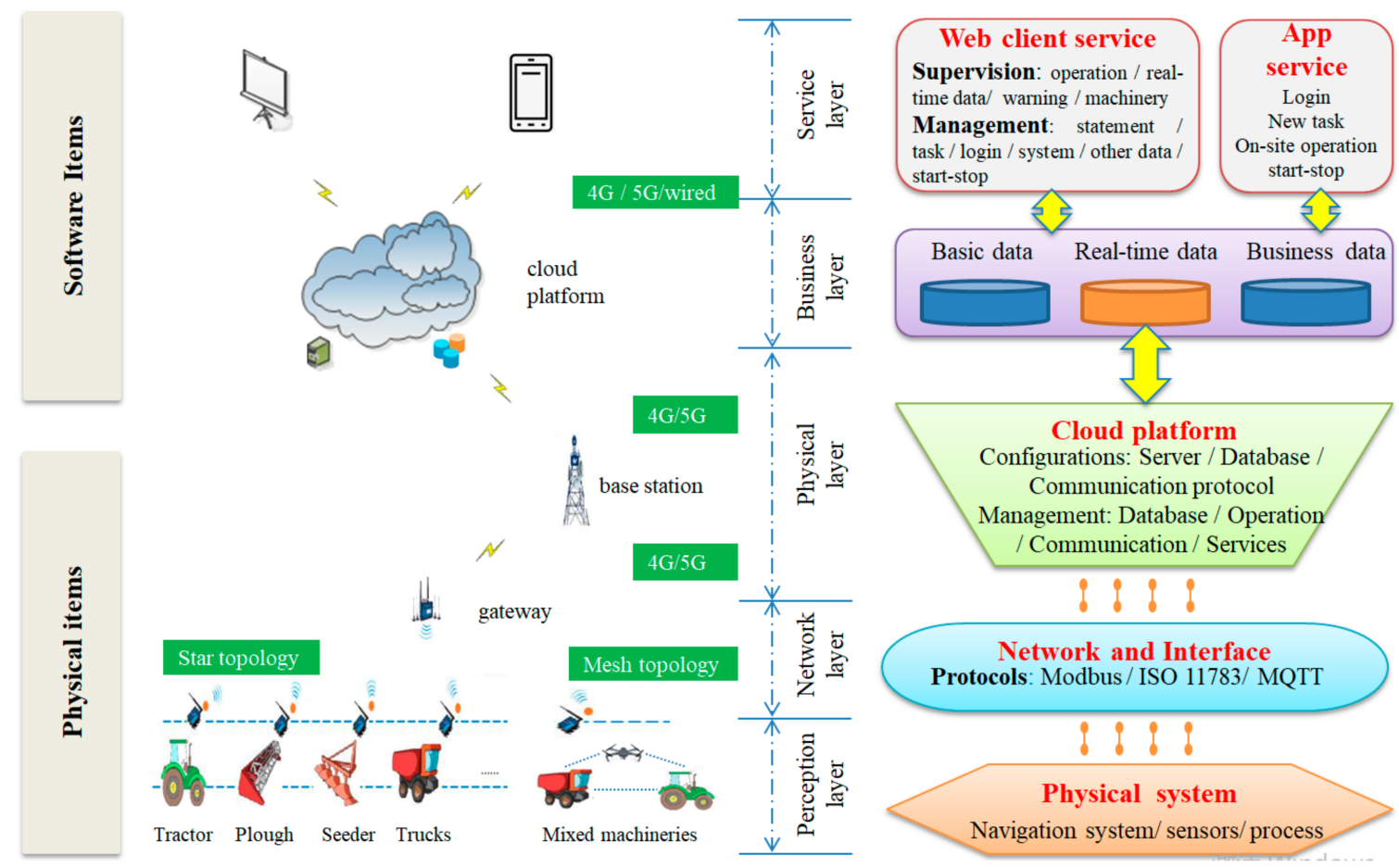

Figure 2. Horizontal integration of AAMMP elements.

\section{Key Enabling Technologies}

\subsection{Multi-Optimization and Cloud Computing}

There are a great many interrelated and inter-restricted problems at the farmland operational scale because of varieties of crops and their related operational processes. In this section, the typical coupling problems of farmland operations are presented, and the multiple objective optimization method is discussed and used to illustrate the functionality of the cloud platform.

\subsubsection{Modeling of AAM Scheduling Problem}

Actual agricultural scale production usually has strong timeliness, especially in the growing seasons, and a series of continuous operations must be completed in a relatively short period of time, such as plowing, seeding, fertilization, and irrigation. A great many agricultural machines participate in these operations and this becomes an AAM scheduling problem. Thus, it is necessary to make a rational plan for each machine in terms of location and function. However, there are also many factors that must be considered, including task make-span, machinery work efficiency, and work path, because of field and task complexity and vehicle size. These typical problems are always coupled and the pursuit of a single optimization goal may lead to lost time and reduced efficiency.

The coupling relationship model of the multi-objective optimization problems at the farmland operational scale is shown in Figure 3. It mainly contains three optimization problems: task allocation for minimizing the make-span, vehicle scheduling for minimizing distances between current locations and fields, and route planning for maximizing field coverage and minimizing operational distances. Two constrained relationships linked with these three problems are also presented, which are static configuration and dynamic regulation. The former involves making sure that all the tasks will be completed using the static optimization approach, and the latter is involved in guaranteeing that the system has a dynamic regulatory capability in case some emergency operations need to be performed preferentially. 


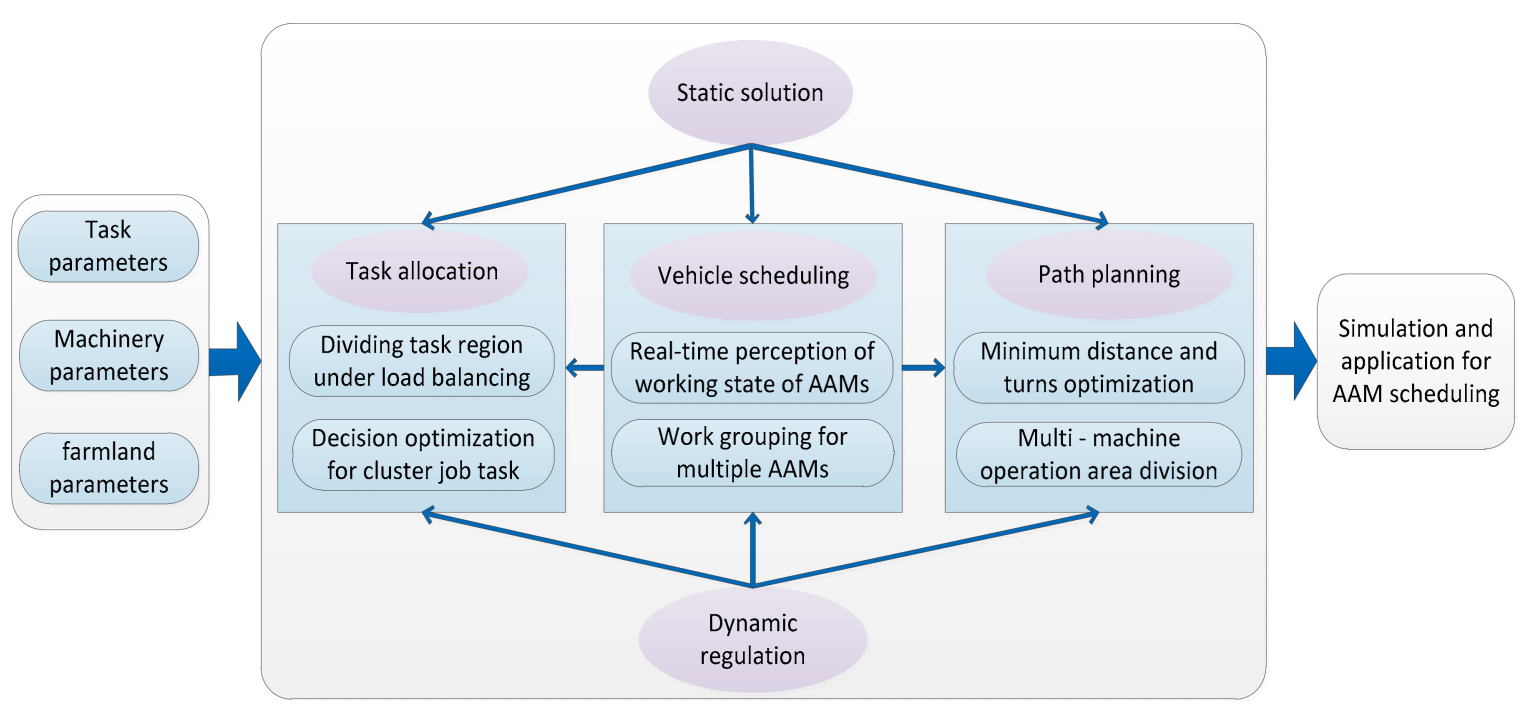

Figure 3. The modeling of the multi-objective optimization problem.

In the AAMMP, task allocation refers to clustering operations at the production scale in accordance with the operation specifications, machinery, time, and other factors. A reasonable operational batch can be formulated by balancing the energy and time consumption. Vehicle scheduling refers to a machinery grouping optimization to minimize the operational time according to the distances between the fields that need to be worked and vehicles capable of carrying out the desired tasks. Usually, the nearest distance method will be adopted to form the machinery team. Path planning involves how the chosen machinery team will work in each field, and the optimization goal is also the minimum time necessary based on the field area and efficiency of the machinery.

Based on the characteristics of the multi-objective optimization problem at the farming scale, a model that takes the total operation time as the optimization goal isformulated; the variables used in the model are defined as Table 2 shows; and the details, including definitions, optimization object, related constraints, parameters, and decision variables, are as follows:

Objective function:

$$
\min T=\max \left\{T_{1}, T_{2}, \ldots, T_{I}\right\}
$$

subject to:

$$
\begin{gathered}
T_{i} \geq \sum_{j, k}\left(T_{i k} \times z_{i j k}\right), \forall i=1, \ldots, I, \forall j=1, \ldots, J, \forall k=1, \ldots, K \\
T_{i k} \geq \operatorname{trans} T_{i k}+\operatorname{ready} T_{i k}+\operatorname{work}_{i k} \\
\sum_{j} U_{i j} \geq Y_{i}, \forall j=1, \ldots, J \\
T_{j k}^{E} \leq T_{i k} \leq T_{j k}^{L} \\
\operatorname{trans} T_{i k} \geq \max \left(\operatorname{trans} T_{i k 1} \times z_{i k 1}, \operatorname{trans} T_{i k 2} \times z_{i k 2}, \ldots, \operatorname{trans} T_{i k J} \times z_{i k J}\right) \\
\operatorname{work}_{i k} \geq\left(S_{i}-\sum_{j}\left(\operatorname{trans} T_{i k}-D_{i j} \times z_{i j} / E_{k}\right) \times E_{k}\right) /\left(\sum_{j} z_{i j k} \times E_{k}\right) \\
\operatorname{trans} T_{i j k}<w o w k T_{i k}
\end{gathered}
$$


Table 2. The variables used in formulating the AAM scheduling problem.

\begin{tabular}{cc}
\hline Notations & Remarks \\
\hline$i$ & Indices for field, $i=1, \ldots, I$ \\
$k$ & Indices for machine, $j=1, \ldots, J$ \\
$T$ & Indices for task and its corresponding machine group, $k=1, \ldots, K$ \\
$T_{i}$ & Total operation time of completing all tasks \\
$T_{i k}$ & Total operation time of competing all tasks in field $i$ \\
$T r a n s T_{i k}$ & Operation time of competing task $k$ in field $i$ \\
$r e a d y T_{i k}$ & Traveling time between field $i$ and machine group $k$ \\
$w o r k T_{i k}$ & Ready time of task $k$ for machine group $k$ in field $i$ \\
$T r a n s T_{i j k}$ & Work time of task $k$ for machine group $k$ in field $i$ \\
$D_{i j}$ & Traveling time between field $i$ and machine $j$ that can do task $k$ \\
$E_{k}$ & Distance between field $i$ and machine $j$ \\
$U_{i j}$ & Operating efficiency of completing task $k$ \\
$T_{j k} E$ & 1 if field $i$ can be assigned to machine $j$ \\
$T_{j k} L$ & 0 otherwise \\
$Y_{i}$ & Earliest arrival time of machine $j$ for task $k$ \\
$z_{i j k}$ & Latest arrival time of machine $j$ for task $k$ \\
$S_{i}$ & Task quantity of field $i$ \\
if $k$ machine $j$ is traveling to field $i$ to execute task $k$ \\
otherwise
\end{tabular}

Table 2 shows the meaning of these variables. In the above mathematical model, the objective function (1) was to minimize the total time of a scaled operational task, and this constraint equals the maximum operational time for completing all the tasks in each field. Constraint (2) indicates the operational time of completing each task $k$ in field $i$, whereas Constraint (3) indicates that the total operational time of task $k$ in field $i$ contains three parts: the traveling time between field $i$ and machine group $k$, the ready time of task $k$ for machine group $k$ in field $i$, and the work time of task $k$ for machine group $k$ in field $i$. Constraint (4) indicates that the quantity of machines traveling to field $i$ is more than the task quantity of field $i$, which also means the quantity of machines that execute each task will be equal or greater than 1 . Constraint (5) verifies that the arrival time of machine group $k$ at field $i$ lies within the field $i$ time window, and also indicates that task $k$ cannot be performed until task $(k-1)$ is complete. Constraint (6) indicates that the travel time of machine group $k$ equals the transfer time of the farthest machine $j$ from field $i$. Constraint (7) indicates that the work time of task $k$ equals the machine group $k$ work in field $i$, and although the arrival time of each machine $j$ belonging to machine group $k$ is different, the completion time of task $k$ will be set to be the same. In addition, Constraints (7) and (8) indicate that if the travel time of machine $j$ to field $i$ is greater than the other machines that have arrived and worked in field $i$, the machine $j$ will not participate in the work performed by machine group $k$.

\subsubsection{Decoupling Algorithm for Solving the Multi-Objective Optimization Problem}

Because of the high interdependence and inter-constraints among the three problems, a distributed optimization strategy was used after acquiring the first operational demands; this is shown in Figure 4. The static configuration scheme executes the decoupling algorithm. However, once some urgent tasks arise, these kinds of demands mix with the former uncompleted operations and require dynamic regulation by re-optimizing the problems. 


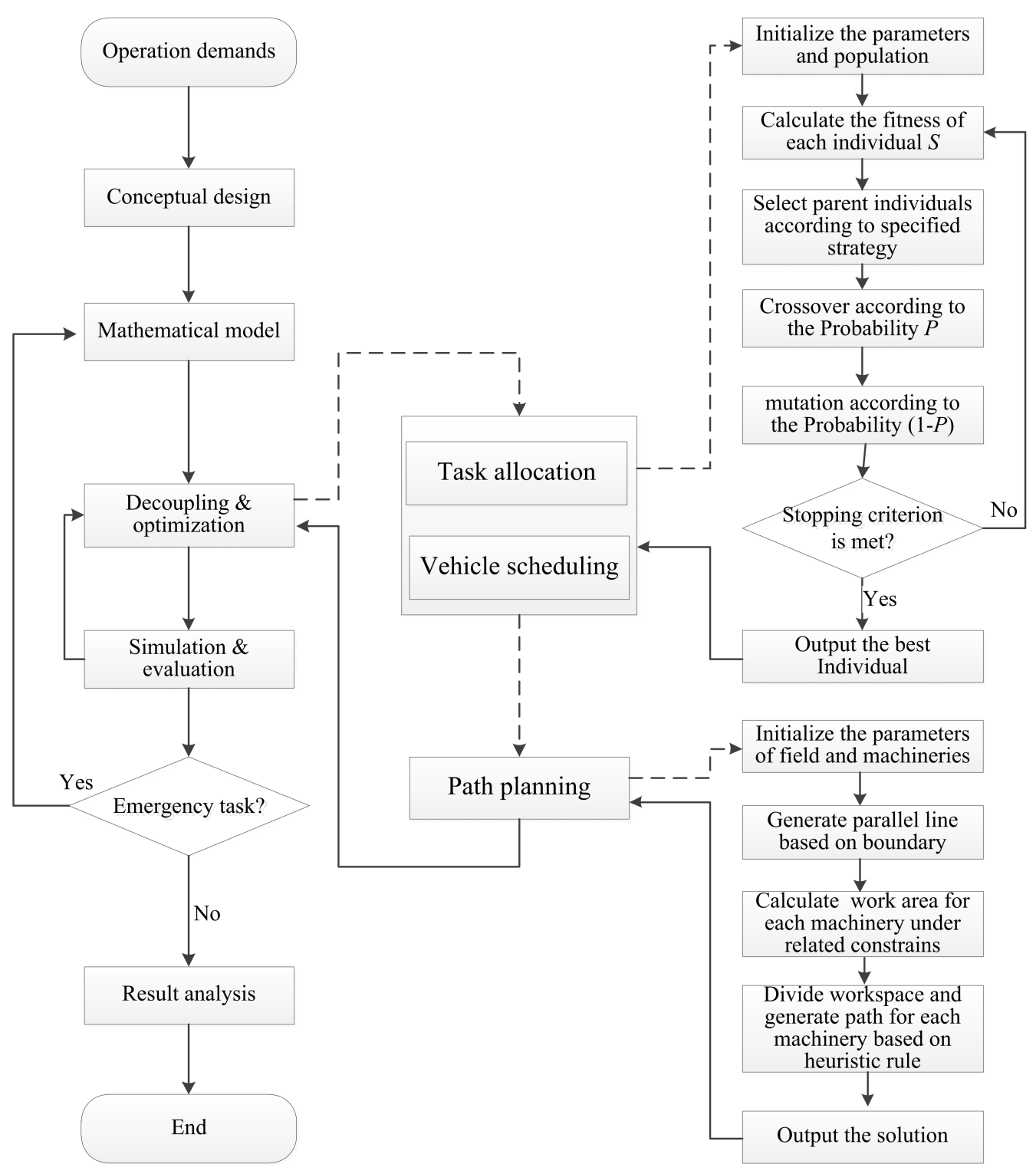

Figure 4. Decoupling algorithm of multi-objective optimization problems.

The proposed decoupling algorithm classifies the three problems into two optimization processes. The first one combines task allocation with vehicle scheduling as a joint optimization in which the model is built with reference to the job-shop scheduling problem. Unlike the job-shop scheduling problem, the migration cost of agricultural machinery from current locations to target plots was also considered, as well as the operational preparation costs after reaching the destination. An improved genetic algorithm (IGA) is used to optimize the operational time and obtain the operational programs. The IGA is encoded with the field ID, and the order in which the ID appears represents the number of tasks in the current field. For example, an order completes the operational tasks of two fields, set as $F_{1}$ and $F_{2}$, and the corresponding task collection is described as $\{(1,2),(2,3)\}$. If a chromosome of the IGA is $\left[F_{1} F_{2} F_{1} F_{2}\right]$, the order of operational tasks will become 1-2-2-3. Then, the corresponding machine group for each task sequence will be scheduled, which is the vehicle scheduling problem. For this problem, we used the greedy strategy based on the shortest distance between the current field and the machines able to perform the desired task to select the number of agricultural machines. A nearby principle and the Constraint (7) and (8) were used to decide if a machine was added to the machine group to complete the current task. 
For path planning, the goal was to decide what route the agricultural machinery will take in the field. After inputting the key parameters, such as the arrival time and equipment width of the machines, as well as the size and shape of the current field, a heuristic method is used to divide the workspace and generate the operational path for each machine. First, a set of parallel lines is generated according to the field boundary and the work width. Then, the work area is divided according to the arrival time of each machine, but the makespan time is set to be the same. In this step, the principle that the first arriving machine will operate in the farthest area is utilized to minimize the operational time. Finally, we generated a series of parallel lines and a full path for each machine under the constraints of their turn radius.

The distributed optimization strategy shown by the decoupling algorithm is separated from the path planning problem, even if the results of this problem affect the value of the fitness. After calculating the operational time according using the Constraint (2) for each field, the total operation time of completing all tasks $T$ can be acquired and then fitness is estimated using Equations (1) and (9), which simplify the calculation and reduced the algorithm complexity.

$$
\text { fitness }=1 / T
$$

A scheduling result worked by four machines in different locations to complete specific assignments in some fields is shown in Figure 5.

In Figure 5, the colored column indicates the operating time in the fields and the white columns before each colored column indicate the total time, which includes the travel time from the field where the assignment was performed to the field to be worked, and the ready time before operation. The original operational plan for five fields is shown in Figure 5a. When all the machines have been working for $30 \mathrm{~h}$, some new tasks, such as the new tasks in fields 6 and 7, need to be performed using this agricultural machinery. Then, the original assignment is dynamically adjusted and the overall undone tasks are rescheduled, as shown in Figure $5 b$.

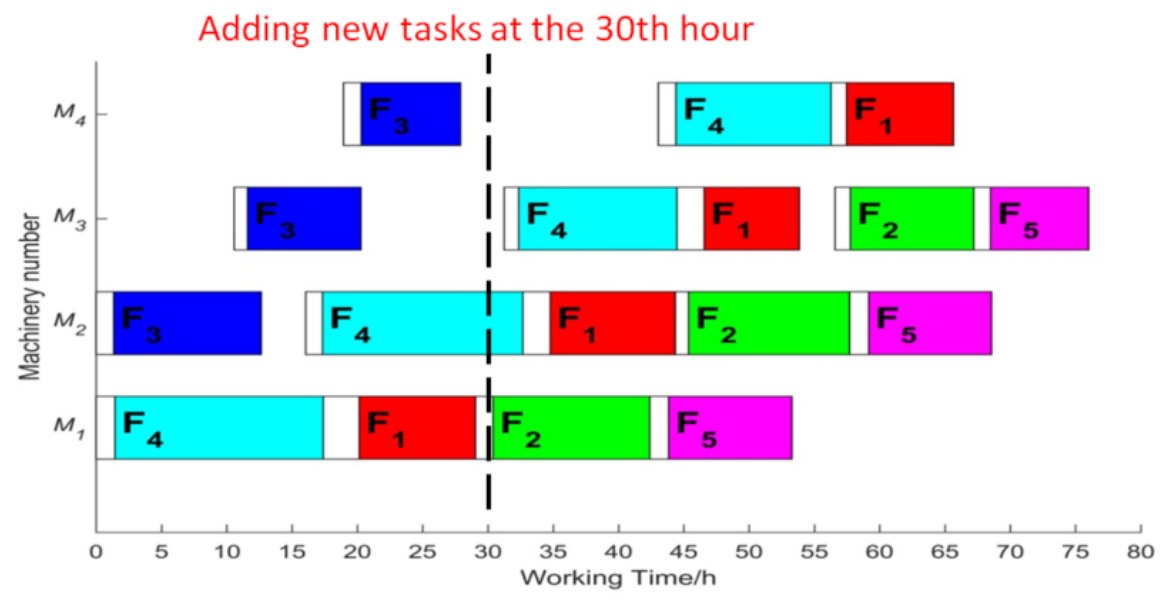

(a)

Figure 5. Cont. 


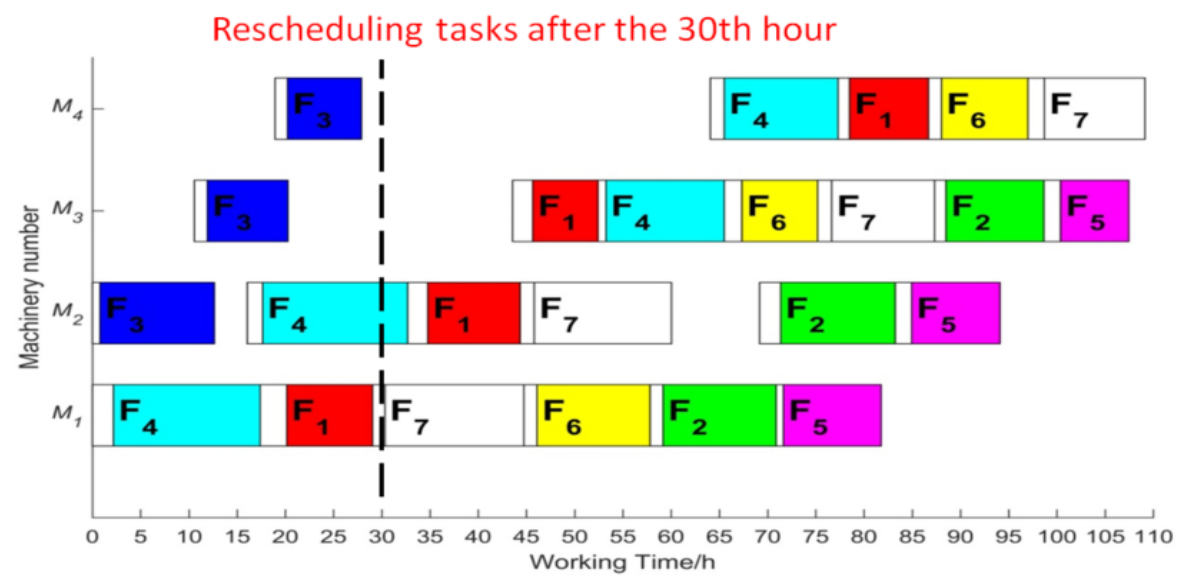

(b)

Figure 5. Illustrations of agricultural machinery allocation schemes: (a) the original operational plan for five fields $\left(\mathrm{F}_{1}-\mathrm{F}_{5}\right)$ executed by four agricultural machines $\left(\mathrm{M}_{1}-\mathrm{M}_{4}\right)$; (b) the dynamic adjustment plan when adding additional tasks in two other fields $\left(\mathrm{F}_{6}, \mathrm{~F}_{7}\right)$ after the 30th hour.

\subsection{System Development}

\subsubsection{Physical Architecture}

As shown in Table 3, the hardware consists of a series of machine tools, including data transfer units (DTUs), embedded systems, and operational machines.

Table 3. Hardware infrastructure of the AMMPS prototype.

\begin{tabular}{|c|c|c|c|c|c|}
\hline Type & Function & Protocol & Devices Name & Devices Details & Photo \\
\hline \multirow{2}{*}{ Data Transfe } & Data Transferring & MQTT & 4G DTU & $\begin{array}{c}\text { YeeCOM Q560 4G } \\
\text { DTU }\end{array}$ & \\
\hline & Protocol Conversing & ISO 11783/Modbus & $\begin{array}{l}\text { Bidirectional } \\
\text { Transducer }\end{array}$ & $\begin{array}{c}\text { ITEKON } \\
\text { CAN232/485 }\end{array}$ & \\
\hline \multirow{2}{*}{ Embedded S } & Navigating & ISO 11783/Modbus & $\begin{array}{c}\text { Automatic } \\
\text { Navigation System }\end{array}$ & Self-Developed & \\
\hline & Motion Controlling & ISO 11783/Modbus & Motion controller & $\begin{array}{c}\text { SonnePower } \\
\text { SPC-STW-2612CMS }\end{array}$ & \\
\hline
\end{tabular}


Table 3. Cont.

\begin{tabular}{|c|c|c|c|c|c|}
\hline Type & Function & Protocol & Devices Name & Devices Details & Photo \\
\hline \multirow{3}{*}{ Operation Machinery } & Tillage/Seeding & Mixed & Tractor & LOVOL M1204-p & \\
\hline & Spraying & Mixed & Sprayer & LOVOL ARBOS & \\
\hline & Harvesting & Mixed & Harvester & LOVOL RG50 & \\
\hline
\end{tabular}

Abbreviations: MQTT, Message Queuing Telemetry Transport; DTU, Data Transfer Unit.

The Data Transfer Unit is comprised of an ITEKON bidirectional transducer and a YeeCOM 4G DTU; the ISO 11783 data from the embedded devices is transferred to the former and translated to Modbus protocol data. It is then sent to the cloud platform by the chosen 4G DTU. The SonnePower motion controller is used to control all of the electronic control units (ECUs) installed in the machinery, such as the throttle position, engine speed, wheel speed, and engine temperature sensors, and the self-developed automatic navigation system enables each agricultural machine to operate in the field automatically. The operational machinery mainly included tractors, sprayers, and harvesters, and all of these machines were produced by LOVOL, a well-known agricultural manufacturing company in China. Both motion controller and automatic navigation systems were installed in each type of machine.

\subsubsection{Software System}

Our research team has studied automatic driving technology in agricultural machinery for the past 10 years, and the team has designed a number of embedded systems for farm machinery automatic driving and unmanned field operations, including systems installed in tractors, rice transplanters, sprayers, and harvesters. The AAMMP was developed based on these embedded systems and an IoT-based service architecture. The development guideline, business processes, and programming languages are unified and they can be integrated and deployed onto a cloud platform.

As shown in Figure 6, a demonstrative prototype AAMMP system was developed by integrating and communicating with the hardware devices. It contained a set of applicationoriented systems involving an app and a web, as well as a cloud platform management system. The first was developed using J2EE technology architecture and integrated with the proposed decoupling algorithm. The others were designed for system configuration and integration, and their functions include system deployment in the cloud server, database connection, communication protocol designation, and remote control of agricultural machinery. In addition, Aliyun, which is part of Alibaba Co., was selected as the server platform. 


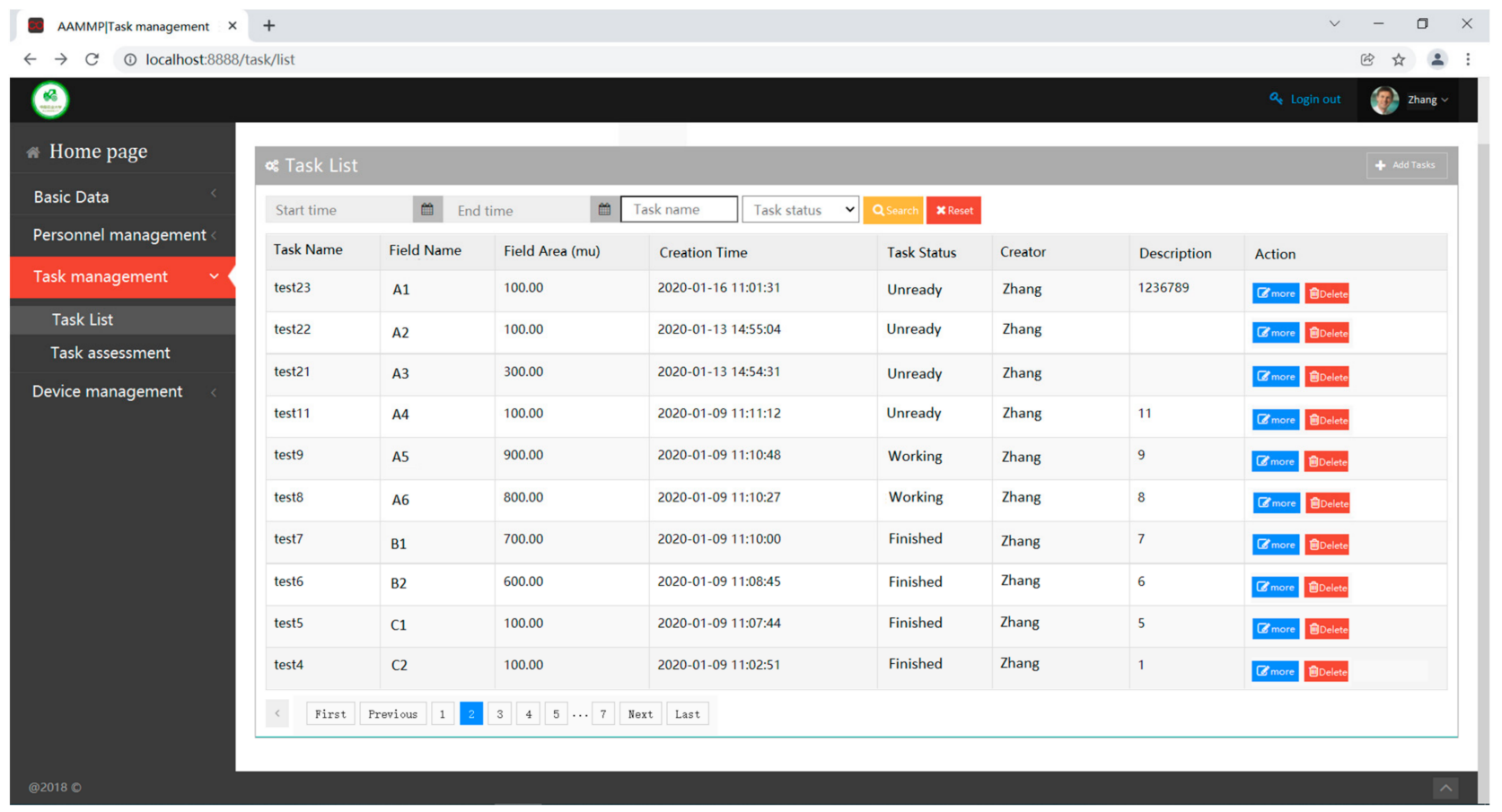

Figure 6. The demonstrative prototype AAMMP system.

\subsection{Network and Communication}

Because of its high reliability and low cost, the Controller-Area-Network (CAN) was used for communication in the chassis systems of the agricultural machines. The ISO 11783 protocol, which includes the CAN 2.0 specifications, and the SAE J1939 protocol have been widely used to transmit data among the ECUs of such chassis systems [34]. To simplify the data processing, we used ISO 11783 based on the CAN bus application layer protocol to transmit data between AAMs and the AAMMP. In the J1939 protocol, the 29-bit identifier of the CAN is physically defined, and the corresponding data definition is formulated. This is implemented and encapsulated by the Protocol Data Unit (PDU). The PDU contains seven sections: priority, retention bit, data page, PDU format, PDU special field, source address, and data field. Thus, the PDU is mainly defined. An example of a data package is present in Figure 7.

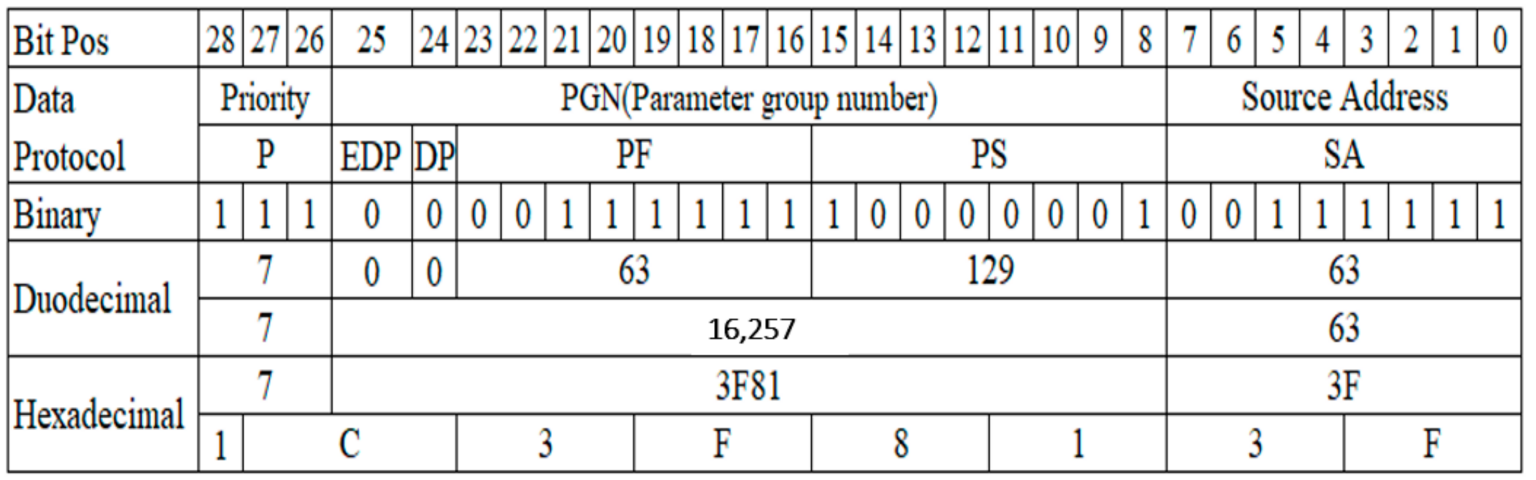

Figure 7. Cont. 


\begin{tabular}{|c|c|c|c|}
\hline \multicolumn{3}{|c|}{ Data Field } \\
\cline { 1 - 1 } Bytes & Variants & Bytes Number & Description \\
\cline { 1 - 1 } B0 & \multirow{2}{*}{ Real-time latitude } & 5 Bytes & $\begin{array}{r}\text { Offset }=-9000000000, \\
\text { Precision }=0.00000001 \\
-90.00000000 \sim 90.00000000\end{array}$ \\
\cline { 1 - 2 } B1 & Course angle & 2 Bytes & $\begin{array}{c}\text { Offset }=0, \text { Precision }=0.1 \\
\text { Range: } 0.0 \sim 360.0, \text { Due east is 0 } 0\end{array}$ \\
\cline { 1 - 2 } B3 & Satellite number & 1 Bytes & Offset $=0, \quad$ RFactor $=10 \sim 255$ \\
\cline { 1 - 2 } B6 & & & \\
\cline { 1 - 2 } B7 & & &
\end{tabular}

(b)

Figure 7. Sample of data package of the proposed protocol. (a) Control field. The PDU (Protocol Data Unit) contains seven sections: priority, retention bit (R), data page (DP), PDU format (PF), PDU special field (PS), source address (SA), and data field. (b) Data field.

We divided all the data into three classes using the defined priority of data transmission. The data with the highest priority include power take-off, start-stop, and mode switch, which are the steering instructions for the agricultural machine to control and execute relative motions. For example, an instruction of 1 will start the tractor running, whereas an instruction of 0 will stop the tractor. The second priority involves the path planning of AAMs, which is calculated in the cloud and then uploaded to the tractor through the proposed network and communication protocol. To ensure that these data are sent and received successfully, after receiving a data package, the receiver will send a feedback data package to the sender, and only when the sender receives the feedback data on a fixed frequency will the data be implemented. This rule ensures that key data is not lost during transmission. The lowest priority is the collection of basic data during AAM operation, including real-time longitude and latitude, altitude, pitch angle, roll angle, identification of satellite signal, operating speed, and total number of satellites.

After the basic protocol between the AAM and agricultural machinery was established, the network and communication link were built, as shown in Figure 8. The automatic navigation system was installed in the agricultural machinery, and it was used to control the automatic operation of the machinery by sending directional commands to the on-board motion controller. The CAN-Modbus translator translated the packaged J1939 protocol data to Modbus-style and then sent it to the 4G DTU. Afterwards, the data acquisition system collected the data package from the 4G DTU and translated it into decimal numbers, which were saved to a database. Then, the AAMMP system could edit and maintain these data using the web client or app.

In order to validate the stability and efficiency of the network, a bidirectional communication performance test was done; the details are interpreted as follows. At first, the package data were sent to the AAMMP from the automatic navigation system installed on the machinery terminal at a frequency of $1 \mathrm{~Hz}$. The experiment was carried out three times, each for one hour. The received data package numbers were 3586, 3590, and 3582, respectively. The average package loss rate was $0.4 \%$, which means the integrity of uploaded data can be guaranteed. Besides, the data sent from the AAMMP to AAM end was about the job task and operational path, and it was usually sent before the job and cached in the navigation system. In our design, these data must be received by AAMs, and a verification strategy of data package loss and re-transmission was formulated to ensure the integrity of data transmission. Therefore, the demand for high real-time performance of the network may be not obvious. Nevertheless, the network latency rate was be tested informally and the result showed there was a certain degree of network delay sometimes, with a maximum delay of $3 \mathrm{~s}$, but the average delay was less than $1 \mathrm{~s}$, which meets the requirements of the system. 


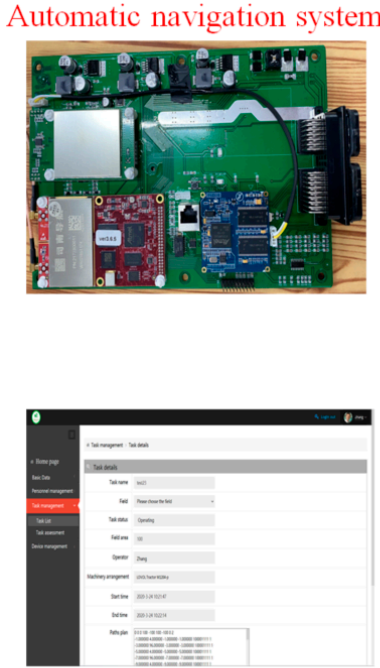

AAMMP web Client
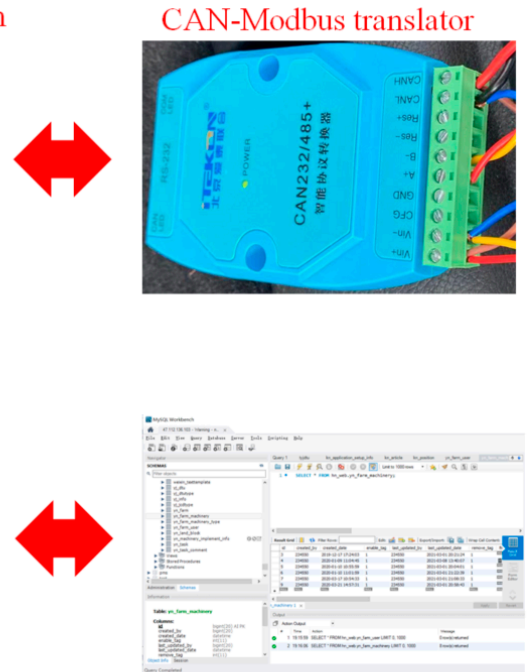

MySQL database

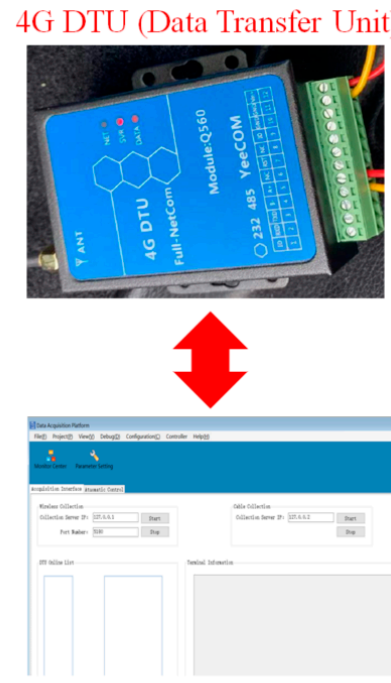

Data acquisition system

Figure 8. The communication system of AAMMP.

\section{Implementation of an AAMMP System}

\subsection{An Illustrative Operational Procedure}

The proposed IoT-based architecture was implemented by integrating the above key techniques, and it is currently being testing and evaluated by our research team. As shown in Figure 9, the implementation effort was conducted in three steps.
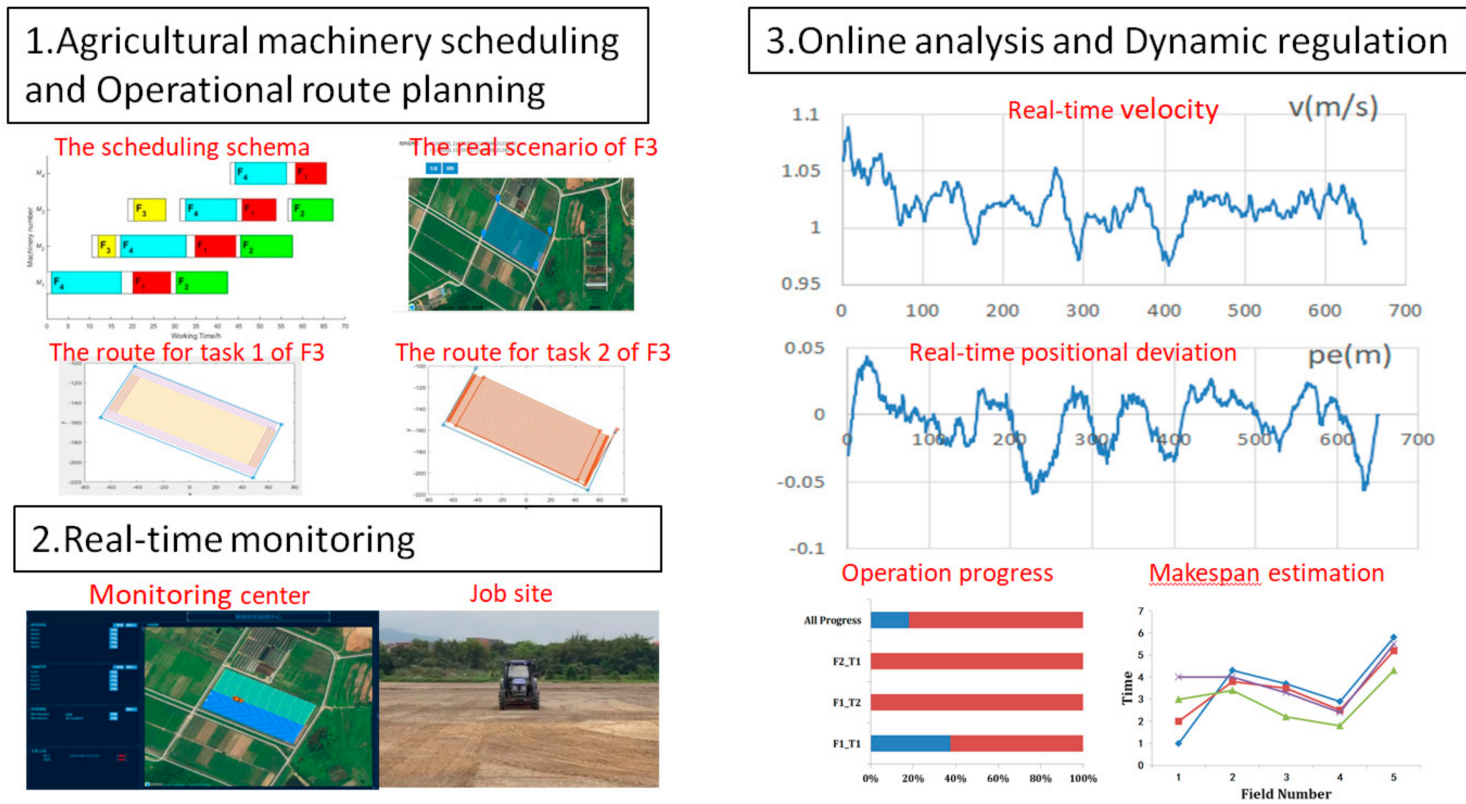

Figure 9. An illustrative AAMMP operational process.

The first step is operational planning. Initially, operational parameters for each individualized task were collected and classified into three types: basic information (e.g., field amount, time limitation, and job type), locational variables, and operational requirements. The information was correlated into control parameters of AAMs, system configuration parameters, predefined models, and algorithms, generating a static execution scheme that corresponds with the agricultural machinery dispatching scheme and operational route for 
each task in each field. Once the planned path is verified using the virtual field model of the AAMMP, it can be fed into the corresponding physical AAM.

The second step is operational monitoring. After deploying the plan to each agricultural machine, the real-time data from the working machines was scanned and analyzed by the AAMMP, enabling the operators and farmers to know the real-time operational status. If something unexpected happens when the machine is operating, such as some emergency tasks that need to be preferentially completed or the breakdown of working machinery, then the scheme will be rebuilt and reallocated to the agricultural machinery, as Figure 6 shows. By enabling peer-to-peer connections between smart agricultural devices and online virtual models at any time and any place, the AAMMP can monitor the operations of multiple machines at the same time.

The last step involves dynamic analysis and adjustment based on an operational quality assessment. After completing an assignment in a field, an operational quality assessment involving some operational results, including work area, real time velocity, positional deviation, and the estimated make-span, was calculated and an operational analysis report was generated. According to the operation efficiency and finish time of each machine, a new set of the most suitable schemes for the remaining tasks may be regulated dynamically and redistributed to the agricultural machinery.

\subsection{System Performance Analysis}

The AAMMP completed three kinds of service for farmers, namely, AAM scheduling, operational route planning and real-time monitoring. These service resources provided by the AAMMP provided significant improvements in many aspects, such as operational efficiency and AAM utilization. With regard to the former, after the farmers use the monitoring center of the AAMMP, all the real-time information on the entire operation process of the whole device can be obtained. Based on this, the decisions made by the operator of AAM in each link are more in line with the operational sites and processes, improving the operational efficiency of all links and reducing operating costs. In terms of the increase of AAM utilization, taking the case of Figure 5 as an example, the number of original tasks is 17 in five fields and the make-span is $76.2 \mathrm{~h}$ (Figure 5a); when the case is executed at $30 \mathrm{~h}$, seven new tasks in two fields are added. By recalculating with the proposed optimization algorithm, the completion time was reduced from $112.8 \mathrm{~h}$ to $108.6 \mathrm{~h}$ by allocating tasks more rationally, saving $4.2 \mathrm{~h}$.

\subsection{Discussion}

The application of the proposed key techniques and the implementation of the AAMMP result in three major benefits. First, engineers can quickly configure an AAMMP, equip the agricultural machines, and integrate the middleware devices because the clear cyber design eliminates many uncertainties. Additionally, using the proposed approach and communication protocol, the implementers can also develop systems and inter-connections without the presence of professional agricultural machinery. Secondly, from the farmer's perspective, the AAMMP is an appropriate tool to manage and arrange their agricultural machines' operations automatically in accordance with the operational tasks, avoiding long-term decisions and reducing labor costs. Moreover, the proposed AAM scheduling model is relatively flexible, owing to the dynamic adjusting operational plan that assesses the real-time working conditions of the agricultural machinery, ensuring that all the tasks can be completed on time. Finally, some operation data uploaded from the AAM to the system can be used for accessing operational quality and can also be combined into large datasets for building AAM data mining models, including fault warning and operational efficiency prediction models.

Three challenges related to the AAMMP should not be ignored. The first is the robustness of the network [22,35]. Stable wireless communication is an important limitation because the harsh $A A M$ working environment may be removed from $4 \mathrm{G} / 5 \mathrm{G}$ signals and wireless infrastructure. The second is data security and privacy. Researchers and developers 
tend to concentrate on sensing, processing, controlling, and computing, with less effort going to handling security threats and privacy, which may lead to attacks on the smart devices and systems [36]. The third is how to improve the inflexibility and interoperability of the IoT-based system. Interoperability is characterized by integrating frameworks, applications, services, and models to work in a combined and stable way [37]. However, sometimes, the large real-time interconnection between the IoT-based system and the agricultural machines may encounter some errors, which may result in a system crash or even machine failure.

\section{Conclusions}

This paper used the smart farm as an implementation scenario and proposed an IoT-enabled cloud management platform to manage and control the operation of agricultural machinery equipped with automatic navigation system. By analyzing configuration parameters and system variables, as well as the logic and processes of how the system worked, a layered IoT-based architecture was used to realize the cooperative operation of multiple agricultural machines in arable farming. The AAM scheduling model benefited from the idea of "iterative optimization between static execution solution and dynamic adjustment strategy", which enhanced the practicability of the system in a real situation. Using a coupling algorithm as the core, the AAMMP was developed and implemented by integrating physical entities and software modules. A CAN-Modbus protocol conversion strategy and a 4G-based network link were proposed to build the bridge between the AAM and the AAMMP, and the communication test proved the reasonableness of these methods. As evidenced from a successful application to perform practical farming tasks, the proposed method provided an optimal guide and the system controlled the agricultural machinery.

The application of the AAMMP brings two kinds of benefits to a farm. On the one hand, farmers can flexibly match or release their agricultural machinery resources to and from practical production in response to dynamic operational demands, which essentially reduces the investment risk and cost in the operation configuration. On the other hand, AAMMP allows farmers to manage their farm operations by acquiring real-time operation and optimized service solutions provided by both hardware devices and software management. Due to the expertise of the service, the farmers can obtain more refined and professional decision-making, which effectively improves management efficiency and reduces the labor force in the production process. Future work will focus on incorporating big data analytics into the IoT-based digital model for the operation and maintenance of a smart farm management system.

Author Contributions: Conceptualization, Z.Z. and X.L.; methodology, F.Z.; software, W.Z.; validation, F.Z., W.Z. and Z.Z.; formal analysis, Y.L.; investigation, B.W.; resources, F.Z. and W.Z.; data curation, F.Z.; writing-original draft preparation, F.Z.; writing-review and editing, W.Z., Z.Z. and Y.L.; visualization, B.W.; supervision, X.L.; project administration, Z.Z.; funding acquisition, Z.Z. All authors have read and agreed to the published version of the manuscript.

Funding: This research was funded by the National Key Research and Development Program of China (no. 2020YFB1709604), National Natural Science Foundation of China (no. 32071914), and Basic and Applied Research Fund of Guangdong Province of China (no. 2019A1515111152).

Institutional Review Board Statement: Not applicable.

Informed Consent Statement: Not applicable.

Data Availability Statement: The data that support the findings of this study are available on request from the corresponding author.

Acknowledgments: The authors gratefully acknowledge the editors and anonymous reviewers for their constructive comments on our manuscript.

Conflicts of Interest: The authors declare no conflict of interest. 


\section{References}

1. Rains, G.C.; Thomas, D.L. Precision Farming: An Introduction; University of Georgia: Griffin, GA, USA, 2009.

2. Shafi, U.; Mumtaz, R.; Garcia-Nieto, J.; Hassan, S.A.; Zaidi, S.A.R.; Iqbal, N. Precision Agriculture Techniques and Practices: From Considerations to Applications. Sensors 2019, 19, 3796. [CrossRef] [PubMed]

3. Sundmaeker, H.; Verdouw, C.; Wolfert, S.; Pérez Freire, L. Internet of Food and Farm 2020. In Digitising the Industry—Internet of Things Connecting Physical, Digital and Virtual Worlds; Vermesan, O., Friess, P., Eds.; River Publishers: Gistrup, Denmark; Delft, The Netherlands, 2016; pp. 129-151.

4. Pang, Z.; Chen, Q.; Han, W.; Zheng, L. Value-centric design of the internet-of-things solution for food supply chain: Value creation, sensor portfolio and information fusion. Inf. Syst. Front. 2015, 17, 289-319. [CrossRef]

5. Sorensen, C.G.; Fountas, S.; Nash, E.; Pesonen, L.; Bochtis, D.; Pedersen, S.M.; Basso, B.; Blackmore, S.B. Conceptual model of a future farm management information system. Comput. Electron. Agric. 2010, 72, 37-47. [CrossRef]

6. Liu, Q.; Zhang, H.; Leng, J.; Chen, X. Digital twin-driven rapid individualised designing of automated flow-shop manufacturing system. Int. J. Prod. Res. 2019, 57, 3903-3919. [CrossRef]

7. Jayaraman, P.P.; Yavari, A.; Georgakopoulos, D.; Morshed, A.; Zaslavsky, A. Internet of Things Platform for Smart Farming: Experiences and Lessons Learnt. Sensors 2016, 16, 1884. [CrossRef]

8. Kosior, K. Digital transformation in the agri-food sector-opportunities and challenges. Annls Pol. Assoc. Agric. Agribus. Econ. 2018, 2, 98-104. [CrossRef]

9. Li, S.; Xu, H.; Ji, Y.; Cao, R.; Zhang, M.; Li, H. Development of a following agricultural machinery automatic navigation system. Comput. Electron. Agric. 2019, 158, 335-344. [CrossRef]

10. Zhao, C.J.; Yang, X.T.; Li, B.; Li, M.; Yan, H. The retrospect and prospect of agricultural information technology in China. J. Agric. 2018, 8, 180-186.

11. Ji, C.Y.; Zhou, J. Current situation of navigation technologies for agricultural machinery. Trans. Chin. Soc. Agric. Mach. 2014, 45, $44-54$.

12. Wolfert, S.; Sorensen, C.A.G.; Goense, D. A Future Internet Collaboration Platform for Safe and Healthy Food from Farm to Fork. In Proceedings of the 2014 Annual SRII Global Conference, San Jose, CA, USA, 23-25 April 2014.

13. Godwin, R.J.; Richards, T.E.; Wood, G.A.; Welsh, J.P.; Knight, S.M. An economic analysis of the potential for precision farming in UK cereal production. Biosyst. Eng. 2003, 84, 533-545. [CrossRef]

14. Nikkila, R.; Seilonen, I.; Koskinen, K. Software architecture for farm management information systems in precision agriculture. Comput. Electron. Agric. 2010, 70, 328-336. [CrossRef]

15. Murakami, E.; Saraiva, A.M.; Ribeiro, L.C.M., Jr.; Cugnasca, C.E.; Hirakawa, A.R.; Correa, P.L.P. An infrastructure for the development of distributed service-oriented information systems for precision agriculture. Comput. Electron. Agric. 2007, 58, 37-48. [CrossRef]

16. Franco, J.D.; Ramirez-Delreal, T.A.; Villanueva, D.; Gárate-García, A.; Armenta-Medina, D. Monitoring of Ocimum basilicum seeds growth with image processing and fuzzy logic techniques based on Cloudino-IoT and FIWARE platforms. Comput. Electron. Agric. 2020, 173, 105389. [CrossRef]

17. Shamshiri, R.R.; Kalantari, F.; Ting, K.C.; Thorp, K.; Hameed, I.A.; Weltzien, C.; Ahmad, D.; Shad, Z.M. Advances in greenhouse automation and controlled environment agriculture: A transition to plant factories and urban agriculture. Int. J. Agric. Biol. Eng. 2018, 11, 1-22. [CrossRef]

18. Harun, A.N.; Mohamed, N.; Ahmad, R.; Rahim, A.R.A.; Ani, N.N. Improved Internet of Things (IoT) monitoring system for growth optimization of Brassica chinensis. Comput. Electron. Agric. 2019, 164, 104836. [CrossRef]

19. Sanjeevi, P.; Prasanna, S.; Kumar, B.S.; Gunasekaran, G.; Alagiri, I.; Anand, R.V. Precision agriculture and farming using Internet of Things based on wireless sensor network. Trans. Emerg. Telecommun. Technol. 2020, 31, e3978. [CrossRef]

20. Ruan, J.; Shi, Y. Monitoring and assessing fruit freshness in IOT-based e-commerce delivery using scenario analysis and interval number approaches. Inf. Sci. 2016, 373, 557-570. [CrossRef]

21. Chaudhary, R.; Pandey, J.R.; Pandey, P.; Chaudhary, P. Case Study of Internet of Things in Area of Agriculture, 'AGCO's Fuse Technology's' ‘Connected Farm Services'. In Proceedings of the 2015 International Conference on Green Computing and Internet of Things(ICGCIoT), Noida, India, 8-10 October 2015; pp. 148-153.

22. Oksanen, T.; Linkolehto, R.; Seilonen, I. Adapting an industrial automation protocol to remote monitoring of mobile agricultural machinery: A combine harvester with IoT. IFAC-PapersOnLine 2016, 49, 127-131. [CrossRef]

23. Zhang, R.; Hao, F.; Sun, X. The design of agricultural machinery service management system based on Internet of Things. Procedia Comput. Sci. 2017, 107, 53-57. [CrossRef]

24. Li, C.; Tang, Y.; Wang, M.; Zhao, X. Agricultural Machinery Information Collection and Operation Based on Data Platform. In Proceedings of the 2018 IEEE International Conference of Safety Produce Informatization (IICSPI), Chongqing, China, 10-12 December 2018; pp. 472-475.

25. Fu, W.; Dong, X.R.; Shuai, W.; Yang, M.; Wang, J. The Intelligent Supervision System of Farm Based on "Internet + BDS + GIS". In International Conference in Communications, Signal Processing, and Systems; Springer: Singapore, 2015; pp. 813-820.

26. Kim, W.-S.; Lee, W.-S.; Kim, Y.-J. A Review of the Applications of the Internet of Things (IoT) for Agricultural Automation. J. Biosyst. Eng. 2020, 45, 385-400. [CrossRef] 
27. Pitakaso, R.; Sethanan, K. Adaptive large neighborhood search for scheduling sugarcane inbound logistics equipment and machinery under a sharing infield resource system. Comput. Electron. Agric. 2019, 158, 313-325. [CrossRef]

28. Cao, R.; Li, S.; Ji, Y.; Zhang, Z.; Xu, H.; Zhang, M.; Li, M.; Li, H. Task assignment of multiple agricultural machinery cooperation based on improved ant colony algorithm. Comput. Electron. Agric. 2021, 182, 10599. [CrossRef]

29. He, P.; Li, J.; Wang, X. Wheat harvest schedule model for agricultural machinery cooperatives considering fragmental farmlands. Comput. Electron. Agric. 2018, 145, 226-234. [CrossRef]

30. Zhang, F.; Luo, X.; Zhang, Z.; He, J.; Zhang, W. Research on Agricultural Machinery Scheduling Problem based on Improved Multi-parents Genetic Algorithm. Trans. Chin. Soc. Agric. Eng. 2021, 37, 192-198.

31. Zhang, F.; Gao, Y.; Li, Y. Research on Cross-Regional Emergency Scheduling and Allocating Strategies. Int. J. Grid Distrib. Comput. 2016, 9, 89-98. [CrossRef]

32. Seyyedhasani, H.; Dvorak, J.S.; Roemmele, E. Routing algorithm selection for field coverage planning based on field shape and fleet size. Comput. Electron. Agric. 2019, 156, 523-529. [CrossRef]

33. Jensen, M.F.; Bochtis, D.; Sørensen, C.G. Coverage planning for capacitated field operations, part II: Optimisation. Biosyst. Eng. 2015, 139, 149-164. [CrossRef]

34. Oksanen, T.; Piirainen, P.; Seilonen, I. Remote access of ISO 11783 process data by using OPC Unified Architecture technology. Comput. Electron. Agric. 2015, 117, 141-148. [CrossRef]

35. Vuran, M.C.; Salam, A.; Wong, R.; Irmak, S. Internet of underground things in precision agriculture: Architecture and technology aspects. Ad. Hoc. Netw. 2018, 81, 160-173. [CrossRef]

36. Tuna, G.; Kogias, D.G.; Gungor, V.C.; Gezer, C.; Tas,kın, E.; Ayday, E. A survey on information security threats and solutions for Machine to Machine (M2M) communications. J. Parallel Distrib. Comput. 2017, 109, 142-154. [CrossRef]

37. Udoh, I.S.; Kotonya, G. Developing IoT applications: Challenges and frameworks. IET Cyber-Phys. Syst. Theory Appl. 2018, 3, 65-72. [CrossRef] 